\title{
Fasten im Ramadan als Herausforderung
}

\begin{abstract}
Für eine optimale medizinische Versorgung von Migranten ist es wichtig, kultur- und migrationsspezifische Bedürfnisse zu beachten. Das gilt v.a. bei muslimischen Diabetikern im Ramadan.
\end{abstract}

Im Ramadan ist das Fasten für alle gesunden erwachsenen Muslime Pflicht, erklärte Dr. Majid Hamdouchi, Allgemeinmediziner in Offenbach. Als Erfüllung einer im Koran offenbarten Verpflichtung diene es dazu, sich seines Glaubens neu bewusst zu werden.

Es sei auch mit Hoffnung auf Vergebung der Sünden verbunden, solle Solidarität mit Armen zeigen und den Körper reinigen, so Hamdouchi. Trotz seiner Vorteile könne Fasten auch bei gesunden Menschen zu gesundheitlichen Proble- men führen, und Kranken ernsthaft schaden. Dazu komme es immer wieder, da kranke Muslime trotz Befreiung von der Verpflichtung am Fasten teilnehmen. Hier ist nach Erfahrung von Hamdouchi Überzeugungsarbeit nötig. Wollen Diabetiker fasten, sollten sie dies nur unter Überwachung eines Arztes tun.

Er empfahl kurz vor Sonnenauf- und bei Sonnenuntergang Nahrungsmittel mit hohem Faseranteil und niedrigem glykämischem Index zu essen. Um den Blutzucker nicht zu steil steigen zu lassen, sollte in der halben Stunde nach Einnahme eines Antidiabetikums Nahrung mit hohem glykämischem Index gemieden werden. Zudem müsse vor und nach den Mahlzeiten Blutzucker gemessen und das Fasten sofort beendet werden, sobald Symptome einer Unterzuckerung auftre-

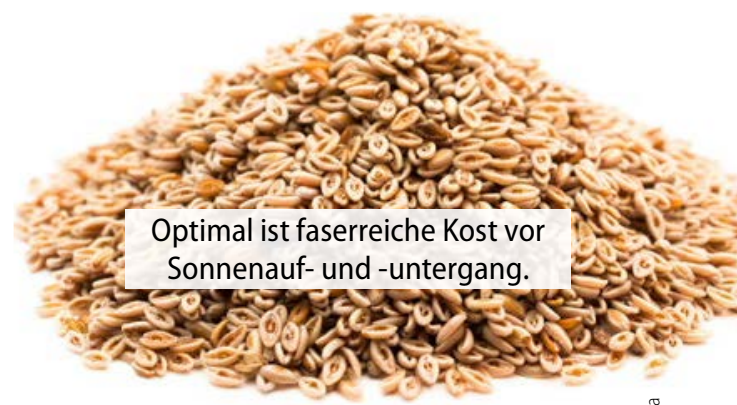

ten, so Hamdouchi. Typ-1-Diabetikern riet er wegen des Ketoazidoserisikos vom Fasten $\mathrm{ab}$.

Um bei Migranten das Gesundheitswissen und die Teilnahme an Vorsorgeund Früherkennungsangeboten zu fördern, müssen Barrieren abgebaut werden, so der Sozialwissenschaftler Ramazan Salman, Geschäftsführer des Ethno-Medizinischen Zentrums e.V. (EMZ), und Begründer des MiMi-Projekts, Hannover. MiMi arbeitet mit mehr als 2.000 transkulturellen Lotsinnen und Mediatoren unter den Migranten. Sie helfen, die Integration in das deutsche Gesundheitssystem zu verbessern und nutzen dabei in Hannover entwickelte Standards, Curricula und Materialien.

InfoDiabetologie 2016; 10 (1):49 als 20 Punkte ein hohes Risiko, in den kommenden zehn Jahren an Diabetes mellitus zu erkranken. Großer Vorteil des Fragebogens ist, dass er sich ohne ärztliches Zutun ausfüllen und auswerten lässt. Natürlich gibt es auch viele andere Fragebögen zum Diabetes-Screening. Schwarz macht darauf aufmerksam, dass der im FINDRISK enthaltene Taillenumfang ein Surrogat für das viszerale Fettdepot ist. Dieses sezerniert mehr als 200 Hormone. Viele davon lösen eine Insulinresistenz aus. Allein der Taillenumfang sagt also schon etwas über das Diabetesrisiko aus. Zudem ist er ein ideales Feedback bei Lebensstil-Interventionen - besser als die Messung des Körpergewichts.

\section{Nichtinvasiver Glukose-Test}

Mit der EZSCAN ${ }^{\mathrm{Tm}}$-Methode scheint es zudem jetzt ein nichtinvasives Verfahren zur Bestimmung der Glukose oder der Insulinresistenz zu geben. Über zwei Fuß- und zwei Handelektroden wird eine geringe Gleichstromspannung angelegt und in Millivoltschritten gesteigert. Mit Hilfe eines mathematischen Algorithmus und basierend auf der reversen Iontopho- rese wird der eingeschränkte Funktionsgrad autonomer Nervenfasern bestimmt. Dieser korreliert direkt mit der Insulinresistenz und einer gegebenenfalls vorhandenen Neuropathie. Die Wahrscheinlichkeit einer metabolischen Störung lässt sich in vier Gruppen von keinem Risiko bis zu einer sehr hohen Wahrscheinlichkeit eines Diabetes mellitus angeben.

Laut Schwarz, der selbst an der Evaluation der Methode beteiligt gewesen ist, kann der Test jederzeit und ohne spezifische Vorbereitungen sowie unabhängig von der Nahrungsaufnahme erfolgen. Er liefere innerhalb von zwei bis drei Minuten ein Ergebnis. Die Resultate klinischer Studien in Deutschland, Indien und den USA seien nahezu identisch. Das Gerät ist in Europa, Nordamerika, Australien und anderen Ländern zugelassen und wird bereits für Screenings, etwa in den Niederlanden, genutzt.

\section{Muskelzellen zur \\ Insulinverstoffwechselung}

Die im Vergleich zur Detektion von Risikopatienten noch anspruchsvollere Aufgabe ist die Vrsorge mit Veränderungen des Lebensstils. „Eine grundsätzliche Prävention des Diabetes mellitus besteht in der Verbesserung der Insulinsensitivität“, schreibt Schwarz. Soll heißen, es müssen mehr Zellen mit mehr Insulinrezeptoren entstehen, also Muskelzellen, die die Glukose verstoffwechseln. Erreicht werden kann das mit körperlicher Bewegung.

Schwarz: „Es ist tatsächlich so, dass Alltagsaktivität im Vergleich zu allen anderen getesteten Mechanismen die erfolgreichste Maßnahme darstellt." 10.000 Schritte pro Tag oder etwa 20 Minuten Hausarbeit oder Spazierengehen nach einer kohlenhydratreichen Mahlzeit erhöhen die Zahl von Insulinrezeptoren auf Muskelzellen, und zwar viel mehr und nachhaltiger als etwa zweimal intensiver Sport pro Woche. „Das Ziel ist damit klar, die Umsetzung einfach, lebensnah und realistisch. "Studien zeigen, dass der Anstieg des Blutglukosespiegels nahezu komplett ausbleibt, wenn sich Patienten im Anschluss an kohlenhydratreiche Mahlzeiten $20 \mathrm{~min}$ normal bewegen (Hausarbeit, Spazierengehen).

(Thomas Meißner) 